\section{Classical interferometry and quantum statistics}

from J. Byrne

IN the half-century since the advent of de Broglie's theory of matter waves many striking and beautiful demonstrations have witnessed to the truth of this hypothesis. Under various shapes and guises the classic experiments of Young and Fresnel have been reproduced with particle beams, the motivation, at least in part, being the desire to determine the limits within which these observations may be encompassed by the framework of a classical wave field. Experiments which differentiate between classical and quantum wave phenomena are rare exceptions, and the interest they excite is correspondingly greater. A recent variant (Werner et al., Phys. Rev. Lett., 35, 1053; 1975) of Young's double slit experiment, which establishes in a direct and unambiguous way the fermion character of neutrons, falls squarely in the rare category-a new departure in a field with a long and distinguished history of innovation.

Angular momentum algebra, the language of rotations, is not a topic which normally rates a mention in treatises on interferometry, but in the present context it provides a useful starting point. The notion of a vector is familiar in classical physics as an entity, which, having magnitude and direction, is characterised by three real numbers. In quantum systems of spin $S$ the number of components is $2 S-1$ and by this criterion vector fields have unit spin. However $S$ is also permitted to take half-integral values; this brings in spinor fields of which two component systems are the most prominent. The spin-statistics relation, which associates integral spin with bosons and halfintegral spin with fermions, rests on the firmest of theoretical and experimental grounds.

For the operation of rotation to have a meaning for spinors the components are necessarily complex; the additional degree of freedom implies that spinors may be assigned a phase in addition to their magnitude and direction. An unavoidable consequence is that spinors undergo a sign change under rotation through an angle of $2 \pi$ about any axis, the property which is of current experimental interest.

Uncharged and having a small magnetic moment, the neutron represents an ideal fermion system in which to detect the phase difference between coherently superposed spinor fields which have been subjected to a relative rotation. (Bernstein, Phys. Rev. Lett., 18, 1102; 1967; Aharonov and Susskind, Phys. Rev., 158, $1237 ; 1967)$. In a magnetic field the two spin states have energies $\pm \frac{1}{2} h \omega_{\mathrm{L}}$, where $\omega_{\mathrm{L}}$ is the angular frequency of Larmor precession. Because the force field is conservative the magnetic energy is compensated by a change in kinetic energy and the free spinor

$$
\mathbf{U}_{\mathrm{o}}=\left(\begin{array}{l}
a_{+} \\
a_{-}
\end{array}\right) \exp \left(i p_{0} x / \hbar\right)
$$

is transformed in a magnetic field to

$$
\begin{gathered}
\mathbf{U}_{\mathbf{H}}-\left(\begin{array}{cc}
a_{+} \exp \left(i \sqrt{ }\left(p_{\mathrm{o}}{ }^{2}-m \hbar \omega_{\mathrm{L}}\right) \cdot x\right) \\
a_{-} \exp \left(i \sqrt{ }\left(p_{\mathrm{o}}{ }^{2}-m \hbar \omega_{\mathrm{L}}\right) \cdot x\right)
\end{array}\right) \\
\simeq\left(\begin{array}{cc}
\exp \left(-i \omega_{\mathrm{L}} x / 2 p_{\mathrm{o}}\right) & 0 \\
0 & \exp \left(-i \omega_{\mathrm{L}} x / 2 p_{\mathrm{o}}\right)
\end{array}\right) \mathbf{U}_{\mathrm{o}}
\end{gathered}
$$

to first order in $\hbar \omega_{\mathrm{L}} /\left(p_{\mathrm{o}}{ }^{2} / 2 m\right)$. The transformation matrix represents a rotation of the spinor through an angle $\varphi=m \omega_{\mathrm{L}} x / p_{\mathrm{o}}$.

The coherent superposition of $\mathbf{U}_{\mathbf{H}}$ and $\mathbf{U}_{\mathrm{o}}$ results in the spinor $\mathbf{U}_{\mathbf{C}}-\mathbf{U}_{\mathbf{H}}+\mathbf{U}_{\mathbf{o}}$ for which the intensity is

$$
I=\left|U_{\mathrm{C}+}\right|^{2}+\left|\mathrm{U}_{\mathrm{C}-}\right|^{2}=4\left(1+\cos ^{2}(\varphi / 2)\right)
$$

The minimum at $\varphi-=2 \pi$, which corresponds to a shift of half a fringe in the interference pattern as $\mathbf{U}_{\mathbf{H}}$ rotates through an angle of $2 \pi$, is a direct consequence of the two-valued nature of spinors. Such an effect would not be observed for vector particles such as the metastable orthohelium atom.

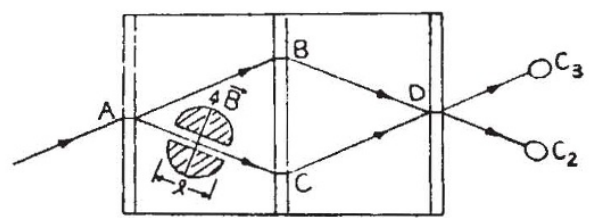

A schematic diagram of the neutron interferometer. On the path $A C$ the neutrons are in a magnetic field $B(0$ to $500 \mathrm{G})$ for a distance $l(2 \mathrm{~cm})$.

The very simplicity of these ideas tends to mask the formidable technical problem presented by a laboratory investigation in which the combination of spatial coherence in the two beams with total spatial separation is of the essence. The system adopted by the experimenters, and illustrated in the figure, bears some resemblance to an optical interferometer of Mach-Zehnder type. A symetrical Laue reflection at $B$ gives two coherent beams with an angular separation of twice the Bragg angle; following further reflections in the mirror $B C$ the beams are recombined in the analyser $A D$. All elements of the interferometer are formed by cutting grooves in a single dislocationfree crystal of silicon. One of the component beams propagates normal to a magnetic field and the resultant interference pattern is observed by recording the counting rates at $\mathrm{C}_{2}$ and $\mathrm{C}_{3}$. The crucial advantage which the interferometer enjoys over its optical counterpart stems from the use of diffraction for purposes of amplitude division which, for small Bragg angles, results in an achromatic system of fringes (Bonse and Hart, Appl. Phys. Lett., 6, 155; 1965). In these circumstances the coherence length is effectively infinite and the Laue technique may be employed in a broad band of neutron energies.

To conclude, two brief observations may be made. First, the rotation of the spinor $\mathbf{U}_{\mathbf{H}}$ may be established experimentally by measuring the polarisation of those neutrons which traverse the magnetic field. On the other hand the interference effect is observed with the spinor $\mathbf{U}_{\mathbf{c}}$ and for these neutrons the polarisation is turned through an angle of $\pi$ not of $2 \pi$. There is therefore no question of making simultaneous observations of the spin rotation and of the phase difference. The second comment refers to the unitary property of the rotation matrix for spin $\frac{1}{2}$ fermions which implies that an equivalent interference effect should be observable for any two-state system, fermion or boson, which is taken through a full cycle of unitary transformations. It must be emphasised however that only in the case of fermions can such a cycle of change be understood as a spatial rotation.

\section{Canary mantle plume unlikely}

from Peter J. Smith

So much attention has been given to the Hawaiian-Emperor island chain in connection wtih the mantle plume-hot spot hypothesis that there is a tendency to forget the other areas in which similar ideas may be put to the test. One example is the group of seven Canary Islands. Not that the Canaries have been overlooked entirely. Morgan (Nature, 230, 42; 1971), for example, located a mantle plume beneath the islands; Burke and Wilson (Nature, 239, 387; 1972) suggested that the islands were formed over a plume active for the past 22 million years; Wilson (Tectonophys., 19, 149; 1973) classified the Canaries among "young hot spots on ocean floors that may be stationary"; and Schmincke (Geol. Soc. Amer. Bull., 84, 634; 1972) concluded that although there were insufficient data to confirm a hot spot origin, there was nevertheless some support for it.

But in spite of this interest, Anguita and Hernan (Earth planet. Sci. Lett., 27,11 ; 1975) claim that, with the possible exception of Schmincke, no one has really examined the nature of the Canary island chain in any great detail. Certainly it has not been subjected to anything like as much study as the Hawaiian islands. The unfortunate result is that the Canary group "has been incorporated to the geo- 